Short report

\title{
Profile of extrahepatic portal venous obstruction among children in Central India
}

\author{
Mayank Jain', Jenisha Jain², Gauri Rao Passi' ${ }^{2}$ Kamna Jain², Shikhar Jain ${ }^{2}$ \\ 'Global Health City, India \\ ${ }^{2}$ Choithram Hospital and Research Centre, India
}

\begin{abstract}
Introduction: There is a paucity of data regarding the clinical profile of children with extrahepatic portal venous obstruction (EHPVO) from Central India.

Material and methods: Retrospective analysis of 30 children with EHPVO treated between 2002 and 2012.

Results: The median age of presentation was 12.5 years (range 5-14 years) and median duration of symptoms was 3.2 years (range $0.3-10$ years). Home delivery was noted in 30 cases (100\%), umbilical sepsis in $8(27 \%)$ and history of cow dung application over the umbilical cord in 2 cases (6.7\%). Twenty-two cases (73.4\%) presented with upper gastrointestinal (GI) bleeding while 18 (60\%) were referred for pancytopenia and/or hypersplenism. The patients underwent a median of 3.2 sessions of endoscopic therapy for varices. Most patients $(19 / 30,63.4 \%)$ dropped out of serial endotherapy due to the paucity of funds. Shunt surgery was performed in 7 patients.

Conclusions: Home delivery and umbilical sepsis are significant risk factors for EHPVO. Most patients present with Gl bleeding and do well on endoscopic treatment/shunt surgery.
\end{abstract}

Key words: portal hypertension, portal vein thrombosis, oesophageal varices.

\section{Address for correspondence}

Mayank Jain, Gleneagles Global Health City, 439 Cheran Nagar, 600100 Chennai, India, phone: 917312365688

e-mail: mayank4670@rediffmail.com

Extrahepatic portal venous obstruction (EHPVO) is an important cause of portal hypertension. It constitutes $68-76 \%$ of portal hypertension in children from developing countries [1]. There is a paucity of data regarding the clinical profile and follow-up of children with EHPVO from Central India. The present study is a retrospective analysis of 30 children who were treated at Choithram Hospital and Research Centre, Indore, between 2002 and 2012. We collected files of all paediatric patients $(<18$ years) with clinical diagnosis of portal hypertension from the medical records section of our hospital. The diagnosis of EHPVO was confirmed by notes regarding clinical presentation and ultrasound abdomen with the hepatoportal Doppler report showing presence of portal cavernoma. A total of 36 cases were collected. However, due to the paucity of data in the files of 6 patients, they were excluded from the final analysis.
Thirty patients formed the study group. The median age of presentation was 12.5 years (range 5-14 years) and median duration of symptoms was 3.2 years (range 0.3 years to 10 years). Home delivery was noted in 30 cases (100\%), umbilical sepsis in $8(27 \%)$ and history of cow dung application over the umbilical cord in 2 cases (6.7\%). Twenty-two cases $(73.4 \%)$ presented with upper gastrointestinal (GI) bleeding while 18 (60\%) were referred for pancytopenia and/or hypersplenism. Ascites and hypoalbuminemia were noted in $8(27 \%)$ and $6(20 \%)$ patients respectively at some point during either presentation or follow-up.

Endoscopic follow-up data were available for 26 patients. Oesophageal varices $(26,100 \%)$, gastric varices $(10,38.5 \%)$ and portal hypertensive gastropathy (6, 20\%)were the common findings. The patients underwent a median of 3.2 sessions of endoscopic therapy for varices (range 1-8 procedures). Most patients 
(19/30, 63.4\%)dropped out of serial endotherapy due to the paucity of funds. Among the rest, obliteration of varices was noted after a median of 4.8 sessions (range 3-7) of endoscopic treatment. Shunt surgery was performed in 7 patients. Distal splenorenal and mesocaval shunts were performed in 4 and 3 cases respectively. Prothrombotic states were assessed in 8 cases. Only 1 patient showed positivity for lupus anticoagulant and also had hyperhomocysteinaemia. Other complications noted were portal biliopathy $(6,20 \%)$, hepatitis B $(2,6.67 \%)$ and superior mesenteric venous thrombosis $(3,10 \%)$.

Several studies from India have studied EHPVO in infants and children. Sarin et al. [2] compared seven studies that assessed the etiology of portal vein thrombosis (PVT) in infants and children. In the majority of the cases, the cause could not be identified. However, direct injury to the umbilical vascular system (omphalitis, umbilical vein catheterization) or intraabdominal and umbilical sepsis appeared to play an important role. Bhandarkar et al. have reported history of home delivery in majority of patients in their study [3]. A few studies suggest a strong association between Bacteroides fragilis infection and PVT. The transient development of anticardiolipin antibodies has been suggested as a pathophysiological link between this infection and PVT [4]. In our patient cohort, home delivery was noted in all cases and history of umbilical sepsis was also quite common.

A prospective ultrasonographic study from India, however, reported that none of the 11 patients with septicaemia or umbilical sepsis developed portal vein thrombosis over a follow-up of 2 years [5]. It is postulated that the untreated and severe umbilical sepsis, which may seen in home deliveries, could result in portal vein thrombosis.

Patients usually present with haematemesis and melena [6]. Anaemia and splenomegaly are other common features of EHPVO with a reduction in cell lines, but hypersplenism is observed only in $5-10 \%$ of patients. Ascites can present transiently in 10-20\% of children following surgery or GI bleeding [7]. Gall bladder varices and gall stones are common $[8,9]$. Portal biliopathy is recognized in $90-100 \%$ of the cases; however, symptoms usually develop in the adult age groups and reflect advanced disease. Decreased growth velocity and short stature is another complication of EHPVO and occurs due to declining hepatotropic growth factors and growth spurts usually observed after shunt surgery [10,11]. Upper GI bleeding was the commonest presentation in the present series. We noted a higher incidence of pancytopenia/ hypersplenism, which may be related to referral bias.
Documented portal biliopathy (abnormal liver function tests with imaging confirmation) was noted in $20 \%$ of cases. There is a paucity of data on growth parameters in our series as the data were collected retrospectively.

The majority of our cases dropped out of the serial endotherapy protocol due to a paucity of funds, and the prothrombotic status could be evaluated only in a few. An interesting observation was hepatitis B infection in two children due to repeated blood transfusion. It has been reported that the frequency of hepatitis B and C in EHPVO is comparable to that in the general population, but is higher in transfused patients from remote areas [12].

To summarise, EHPVO in an important cause of portal hypertension in children in Central India. Home delivery and umbilical sepsis are common risk factors. Upper GI bleeding is the commonest presentation. Endoscopic treatment is beneficial in the majority of cases. Portal biliopathy and hepatitis B infection are common morbidities noted in these patients. Prospective, long-term follow-up studies would be more fruitful in assessing the profile and risk factors of EHPVO in children.

\section{Disclosure}

Authors report no conflict of interest.

\section{References}

1. Peter L, Dadhich SK, Yachha SK. Clinical and laboratory differentiation of cirrhosis and extrahepatic portal venous obstruction in children. J Gastroenterol Hepatol 2003; 18: 185-189.

2. Sarin SK, Agarwal SR. Extrahepatic portal vein obstruction. Semin Liver Dis 2002; 22: 43-58.

3. Bhandarkar PV, Sreenivasa D, Mistry FP, et al. Profile of extrahepatic portal venous obstruction in Mumbai. J Assoc Physicians India 1999; 47: 791-794.

4. Ni YH, Wang NC, Peng MY, et al. Bacteroides fragilis bacteremia associated with portal vein and superior mesentery vein thrombosis secondary to antithrombin III and protein $\mathrm{C}$ deficiency: A case report. J Microbiol Immunol Infect 2002; 35: 255-258.

5. Yadav S, Dutta AK, Sarin SK. Do umbilical vein catheterization and sepsis lead to portal vein thrombosis? A prospective, clinical and sonographic evaluation. J Pediatr Gastroenterol Nutr 1993; 17: 392-396.

6. Chawla Y, Dilawari JB, Katariya S. Gallbladder varices in portal vein thrombosis. AJR Am J Roentgenol 1994; 162: 643-645.

7. Chiu B, Superina R. Extrahepatic portal vein thrombosis is associated with an increased incidence of cholelithiasis. J Pediatr Surg 2004; 39: 1059-1061.

8. Khuroo MS, Yattoo GN, Zargar SA, et al. Biliary abnormalities associated with extrahepatic portal venous obstruction. Hepatology 1993; 17: 807-813.

9. Bhatia V, Jain AK, Sarin SK. Choledocholithiasis associated with portal biliopathy in patients with extrahepatic portal vein 
obstruction: Management with endoscopic sphincterotomy. Gastrointest Endosc 1995; 42: 178-181.

10. Mehrotra RN, Bhatia V, Dabadghao P, et al. Extrahepatic portal vein obstruction in children: Anthropometry, growth hormone, and insulin-like growth factor I. J Pediatr Gastroenterol Nutr 1997; 25: 520-523.

11. Kato T, Romero R, Koutouby R, et al. Portosystemic shunting in children during the era of endoscopic therapy: Improved postoperative growth parameters. J Pediatr Gastroenterol Nutr 2000; 30: 419-425.

12. Pande C, Kumar A, Sarin SK. Non-cirrhotic portal fibrosis: a clinical profile of 366 patients. Am J Gastroenterol 2006; 101: 191. 